\title{
La escritura de la memoria sanitaria: el Libro de inhumaciones del cementerio inglés de Málaga en la segunda mitad del siglo XIX
}

\author{
The Writing of Health's Memory: The Burial Register of the English \\ Cemetery in Malaga During the Second Half of the 19th Century
}

\author{
Alicia Marchant Rivera* \\ Universidad de Málaga \\ http://orcid.org/0000-0002-1153-1734 \\ amr@uma.es \\ Ana Barrena Gómez \\ Universidad de Málaga \\ http:/ / orcid.org/0000-0001-5246-9327 \\ a.bg@uma.es
}

Recibido: 06/06/2020; Revisado: 04/08/2020; Aceptado: 22/10/2020

\begin{abstract}
Resumen
El presente artículo se centra en el análisis del Libro de inhumaciones del Cementerio Inglés de Málaga, concretamente en los registros de decesos correspondientes al siglo XIX. La comunidad británica residente en la ciudad durante este periodo se erige en un grupo cohesionado dentro de la miscelánea social y económica de la urbe. Se prestará especial atención a las causas de muerte registradas en la unidad documental, para contribuir a la construcción de la historia sanitaria de la ciudad. Para ello nos serviremos de la precisión informativa del registro de estos decesos, al cuidado de los sucesivos cónsules británicos, que constituyen una escritura complementaria y paralela a la escritura última incisa en las lápidas del recinto sagrado.
\end{abstract}

Palabras clave: Libro de inhumaciones, Cementerio inglés de Málaga, siglo XIX, patologías, historia de la sanidad.

*Autora de correspondencia / Corresponding author.

Copyright: @ 2021 ULPGC. Este es un artículo de acceso abierto distribuido bajo los términos de la licencia Creative Commons Atribución-NoComercial-SinDerivar (by-nc-nd) Spain 3.0. 
La escritura de la memoria sanitaria: el Libro de inhumaciones del cementerio inglés...

\begin{abstract}
This article analyses the burial register of the English Cemetery in Malaga, focusing on records of deaths during the nineteenth century. The city's British residents of this period formed a cohesive group within the social and economic miscellany of the city. The causes of death recorded in the documentation have required particular attention in order to help reconstruct the history of health in the city. To this end, we use the informational precision of these death records, kept in the care of successive British consuls, which constitute a text supplementary and parallel to the closing texts inscribed on the tombstones of this sacred enclosure.
\end{abstract}

Key words: Burial Register, English Cemetery in Malaga, 19th Century, Pathologies, History of Health.

\title{
1. INTRODUCCIÓN
}

El Cementerio Inglés de Málaga es el primer solar de enterramiento no católico surgido en la península ibérica, en el año de 1831, gracias a los esfuerzos del cónsul inglés William Mark, quien obtuvo del gobierno civil de la ciudad un terreno en el antiguo camino de Vélez donde ofrecer un entierro digno a sus compatriotas. Sus cuerpos, hasta entonces, se abandonaban con nocturnidad sin recibir el debido culto en las cercanas playas de la Malagueta. A lo largo de casi dos centurias, los sucesivos cónsules británicos administraron el cementerio con una pequeña aportación del gobierno de Gran Bretaña, aportación que fue retirada en 1903. Esto provocó que paulatinamente el mantenimiento del camposanto y sus jardines fuese caminando hasta su práctico abandono en las postrimerías del siglo $\mathrm{xX}$.

El vacío legal que envolvía a la necrópolis propició que el cónsul inglés Bruce McIntyre decidiera traspasar los terrenos a una fundación para lograr financiación y poner en valor el monumento. En 2006, la propiedad del cementerio recayó en manos de la Fundación Cementerio Inglés de Málaga, establecida para mantenerlo y administrarlo como parte del legado histórico de la ciudad. En 2012, le fue otorgada la condición de Bien de Interés Cultural por la Junta de Andalucía y, hoy en día, está registrado en la Asociación de Cementerios Significativos de Europa debido a su gran valor artístico, histórico, literario y botánico. Personajes como los escritores Jorge Guillén, Gerald Brenan, el autor finlandés Aarne Haapakoski o la hispanista Marjorie Grice-Hutchinson, entre otros, se encuentran inhumados en este camposanto (MARCHANT, 2012).

Centrándonos en la documentación que, a día de hoy, se conserva relacionada con el Cementerio Inglés de la ciudad de Málaga, sobresale el Libro de inhumaciones del camposanto, unidad documental que aglutina información relativa a los inhumados en este camposanto durante una horquilla cronológica de 129 años. El Libro de inhumaciones del Cementerio Inglés de Málaga ha sido custodiado personalmente por los sucesivos cónsules británicos en la ciudad desde el año de su fundación, 1831, y, por consiguiente, del primer enterramiento. Hasta que, en 2014, tras examinar las garantías de conservación y consulta que ofrecían las 
distintas instituciones archivísticas de la ciudad, la Fundación Cementerio Inglés de Málaga lo cedió al Ayuntamiento de la misma, en cuyo Archivo Municipal hoy se localiza.

El presente estudio se centrará en el análisis de los primeros 69 años de registro de decesos, es decir, los correspondientes al siglo XIX, ya que la comunidad británica residente durante esta etapa en la ciudad de Málaga manifiesta una serie de características que la perfilan como un conjunto coherente en el seno del mosaico social y económico de la urbe. Marineros, comerciantes y emprendedores, en su mayoría, vinculados al fluctuante ritmo económico de la Málaga de la segunda mitad del XIX. Y los denominados en la literatura de viajes como invalids, aquellos enfermos que vinieron a la ciudad en busca de las benignas condiciones climáticas para la mejoría o recuperación de su dolencia, en las décadas finales del siglo XIX, coincidiendo con el proceso de desindustrialización malagueño. Grupos sociales foráneos, afincados o visitantes, a los que la muerte les sorprendería en la ciudad.

Precisamente, prestaremos una especial atención a las causas de muerte registradas en las anotaciones del Libro, en aras de contribuir a la construcción e identificación de la historia sanitaria de la ciudad. Frente a la parcialidad en el registro de patologías presente en otras unidades documentales coetáneas, como los partes de enfermos del Hospital Noble de la ciudad, la precisión informativa de las reflejadas en estas notas de registro, casi siempre al cuidado de la mano de los sucesivos cónsules británicos, nos conducirá a una radiografía más certera de la realidad sanitaria de la urbe.

A lo largo de las siguientes páginas, pues, se procurará un acercamiento a esta tipología documental, se prestará atención a la escritura paralela y complementaria de esa escritura última de la piedra del camposanto y, sobre todo, a la información histórico-social que de estos registros de decesos se pueda lograr extraer. Teniendo en cuenta que parte de la documentación generada por instituciones hospitalarias públicas o privadas de la ciudad no ha llegado a nuestros días, este Libro de inhumaciones ofrece una singularidad documental que permite forjar una idea sobre el contexto económico, social y sanitario de la capital malacitana y sus núcleos poblacionales no autóctonos durante la segunda mitad del siglo XIX.

\section{LA SALUBRIDAD EN MÁLAGA DURANTE LA SEGUNDA MITAD DEL SIGLO XIX: LA PERSPECTIVA DE LA LITERATURA DE VIAJES}

La ciudad de Málaga durante el siglo XIX experimenta una serie de cambios vinculados al auge y esplendor industrial que empieza a emerger en ella. Este hecho desemboca en una modernización en diversos puntos de la ciudad, y este concepto está ligado a uno más importante y no indisoluble como es la higienización. No se podía concebir la ciudad sin limpieza, abastecimientos de agua, una correcta comercialización de sus alimentos, así como la mejora de los focos insalubres: los mataderos, los hospitales o los cementerios (CAstellanos, 1998: 209-214). 
Ligado a esto último podemos comprobar que desde la centuria anterior ya se iban formulando estas pesquisas en torno a la higiene y la salud. En el año 1787, durante el reinado de Carlos III, se promulga una Real Orden en la que se establece la creación de camposantos fuera de las ciudades, con la consiguiente prohibición de realizar inhumaciones tanto en el interior como en las cercanías de las iglesias (GALANTE, 1990: 603-604). Este hecho responde principalmente a la necesidad de una mejora higiénica, ya que la salud pública de los ciudadanos se encontraba en riesgo por los enterramientos parroquiales en los diversos puntos estratégicos de las ciudades (BRITO, 2015: 237-238).

A raíz de esta medida, las inhumaciones se empiezan a realizar en cementerios situados a las afueras de la ciudad, pero el problema latente no estaba solventado, y este venía de la mano de los viajeros extranjeros o de los foráneos residentes que fallecían en la ciudad. En Málaga, desde finales del siglo XVIII, se produce un incremento de extranjeros que llegan a la ciudad, fomentado por la mejora de los transportes, las vías de comunicación y los alojamientos (FreIRE, 2012: 68). Las razones derivadas de los viajes son muchas, especialmente comerciales, turísticas y también vinculadas al plano de la salud. Estos últimos serían conocidos como viajeros-residentes enfermos o convalecientes o invalids (RuIz, 2008: 176). Es por ello que, a final de esta centuria, los extranjeros representan entre el $8-10 \%$ de la población malagueña (VILLAR, 1982: 207).

A pesar de las vicisitudes por las que atraviesa la comunidad extranjera de Málaga en las primeras décadas del siglo XIX, podemos constatar que para el año 1844 en concreto, se registran en la ciudad 1.057 extranjeros. De estos, 227 eran de origen británico, conformando la segunda colonia extranjera del momento (Rodríguez, 2005: 23-24; KRAUel, 1986: 72-102). La unión de estos viajeros con la población foránea será tan fuerte que llegará un momento en el que sea difícil averiguar las diferencias establecidas entre unos y otros (BURGOS, 1974: 51).

Pero el destino final de estos extranjeros de culto no católico evidencia la cruel realidad de la intolerancia religiosa y de las antiguas afrentas llevadas a cabo en Europa durante las guerras de religión. Muestra de ello es el diverso y elevado número de lugares empleados para la inhumación de los cuerpos:

La situacion, estension y demas circunstancias de los sitios destinados á las inhumaciones de los cadáveres, tienen tanta influencia en la salubridad de una poblacion que es menester mucho cuidado al establecerlos. Varios han sido en Málaga, los sitios dedicados á este objeto (...) Asi que se ha enterrado en la playa, tanto del lado de Levante como del Poniente; en los tejares, en las iglesias, en cuyos algunos de estos parages se conservan muestras todavia que marcan su anterior destino, ó escavaciones accidentales las reveláran (MARTínEZ, 1852: 302).

Las playas malagueñas abrazaron los cuerpos inertes de estos protestantes que no tenían derecho a una inhumación digna. El procedimiento se realizaba de madrugada, siendo el cuerpo enterrado en una fosa vertical, con la cabeza al descubierto mirando al mar, quedando a la intemperie sin ningún tipo de señalización y a la merced de cualquier alimaña que pasase por el paraje (GIRón y 
Arenas, 2003: 359-360; Grice-Hutchinson, 1989: 15-17).

Para solventar dicho problema, sería el Cónsul Británico William Mark el que se encargara durante 7 años de llevar a cabo las gestiones pertinentes para que los protestantes tuvieran un lugar donde descansar y ser visitados por familiares y amigos. El Cementerio Inglés de Málaga ve la luz tras la Real Orden de abril de 1830, convirtiéndose en el primer cementerio protestante de la península ibérica (MARCHANT, 2014: 31). La fuerza y tesón del cónsul británico, unido al buen quehacer del gobernador de la ciudad, el General José Manso, encargado de convocar a la Junta de Sanidad, permitieron la delimitación de este nuevo camposanto en la ladera del camino de Vélez-Málaga, un terreno idóneo con vistas al mar para albergar la morada de los extranjeros de la ciudad (RoDRíGUEZ, 2005: 27-28). De manos del gobernador de la ciudad, llegarían las siguientes palabras recogidas por Marjorie GRICE-HuTCHINSON (1989: 36) en su obra: «Señor cónsul, se tomarán las medidas necesarias para la cesión de un terreno destinado a inhumar decentemente a sus muertos».

En febrero de 1843, el historiador irlandés Martin HaverTy llega a Málaga y será en su obra Wandering in Spain in 1843 donde encontremos una descripción del recinto de enterramiento, así como el origen del mismo, su epigrafía o la botánica, entre otros elementos. Sobre la salud en Málaga, refiere que por las condiciones de la ciudad apenas se desarrollan las enfermedades epidémicas, que la mortalidad apenas llega a superar el 1\%, así como destaca la longevidad que presentan los malagueños (HAVERTY, 1844: 81). Gran parte de la información mostrada en esta obra deriva del informe creado por el cónsul fundador, William Mark, del cual conservamos un fragmento que reproduce el Dr. FrANCIS en Change of Climate (1853: 181):

The salubrity of Malaga is surprising and scarcely credible. The population is dense, there is a great deal of misery; the prisons are crowded, and with a depôt of convicts (about six thousand), a badly conducted police, the wonder must be that it is not a sink of pestilence. Instead of that it is, under all these and other circumstances equally unfavourable, the healthiest place perhaps in the world. I speak from thirteen years'experience. Sometimes two and three days pass without a single death throughout the city.

Louise Mary Anne TENISON, en su obra Castile and Andalucia, adjunta una descripción del cementerio inglés en la que añade las siguientes líneas sobre la llegada de viajeros en búsqueda de la salud, los cuales llegan a conformar una amalgama vital con las familias protestantes que residen permanentemente en la ciudad:

These are several Protestant families permanently residing in the town; artisans employed in the iron foundries, \&c.; and these, added to the numerous visitors, who now flock there in the winter for health, form a very respectable congregation (TENISON, 1853: 17)

En las páginas subsiguientes (TENISON, 1853: 24), vuelve a hacer una alusión 
La escritura de la memoria sanitaria: el Libro de inhumaciones del cementerio inglés...

a los invalids, destacando el papel de Málaga como uno de sus destinos favoritos por su clima suave:

The last few years Málaga has become a very favourite residence for invalids. Its climate, certainly, is exceedingly mild and genial; and the invalid who can obtain rooms facing the sun will seldom suffer from cold during the winter.

El médico inglés Edwin LeE será quien nos aporte una información más precisa sobre el desarrollo sanitario de la Málaga decimonónica. Realiza una descripción completa del Hospital General, en la que pormenoriza su estructura y funcionamiento, siendo crítico con las deficiencias que este presenta. Por otro lado, nos participa los sanitarios que tratarán a los extranjeros. El primero, el Dr. Shortiff, médico inglés afincado en Málaga y adaptado a su vida y costumbres; el Dr. Giraldés y el Dr. Martínez y Montes, ${ }^{1}$ facultativo del Hospital militar. Estos últimos tendrán más contacto con los invalids por su dominio de la lengua inglesa. En cuanto a las dolencias que más se refieren en los consultorios indica que son frecuentes el reumatismo, las fiebres tifoideas y las afecciones catarrales (LEE, 1854: 60). Sobre el Cementerio Inglés, alude al momento exacto de la construcción de la capilla y a la celebración del oficio por parte del reverendo Mr. Brereton, clérigo establecido en la ciudad tras venir a curarse de una dolencia asmática (LEE, 1854: 57-58; MARCHANT, 2014: 50).

En ese mismo año, Mr. Brereton publica una recopilación de los sermones pronunciados durante el bienio 1852-1854. En el prólogo de la obra, hace referencia al clima benigno de la ciudad de Málaga, tan solicitado por los invalids, sobre todo en los meses de invierno, y al contexto en el que el capellán protestante en la ciudad entra en contacto con el sufrimiento corporal y espiritual que se genera entre las propias personas enfermas y los amigos que los acompañan. Con esta razón de base argumenta la peculiar orientación de sus sermones, dirigidos a confortar a los débiles y tristes y a recordar estos temas a los que tienen mayor fortaleza de cuerpo y alma (BRERETON, 1854: V-VI).

Quizás la descripción más directa sobre el camposanto y las enfermedades de los inhumados emana de la pluma del médico Thomas More MADDEN. En On Change of Climate. In the Treatment of Chronic Diseases..., dedica dos capítulos a Málaga, el primero de los cuales lo redacta a su llegada a la ciudad por vez primera en el año 1858. De hecho, los datos que señala en ambos capítulos son fruto de la observación y supervisión de los pacientes que están a su cargo, así como de otros invalids que llegan a la ciudad con la finalidad de recuperar su salud. Subraya

1 Vicente Martínez y Montes (1810-1893) es uno de los personajes de gran transcendencia en la vida cultural y científica del siglo XIX. Granadino de nacimiento, se afinca en Málaga emparentando por matrimonio con una de las familias de la alta burguesía malagueña, los Loring Oyarzabal. Como profesión ejercerá la medicina en el contexto castrense, puesto que era militar. En el año 1845 funda el Instituto Médico Malagueño, así como la Academia de Ciencias Naturales y Exactas de la ciudad. También será Socio Corresponsal de las Academias de Medicina y Cirugía de Madrid y Granada, presidente de la Sociedad Económica de Amigos del País y director de la Caja de Ahorros y Monte de Piedad de Málaga. La obra de gran trascendencia que publicó será Topografía Médica de Málaga. Esta será consultada y citada por los médicos extranjeros que visitan la ciudad, con el fin de justificar los beneficios climáticos que representa Málaga en aras de la recuperación de la salud. 
en su relato que el camposanto alberga los cadáveres de un elevado número de víctimas mortales por tisis, los cuales están en deuda con los esfuerzos del difunto Capitán Mark (William), el padre del cónsul del momento, por haberles otorgado este último lugar de descanso. Y recuerda cómo el rito cristiano de enterramiento se le negaba en Málaga a los protestantes. Igualmente refiere cómo el capellán protestante del Consulado Británico ya había oficiado funerales en el Cementerio Inglés como el del Dr. Joseph Noble, que había muerto de la epidemia de cólera en la ciudad de Málaga (More, 1874: 136-137).

Cuando un extranjero fallece fuera de su tierra, son las autoridades locales junto con el cónsul del país los que se encargan de hacer las gestiones pertinentes para informar a los familiares o allegados de la pérdida del ser querido. La viajera Lady Sophia DunBar refleja en A family tour round the coasts of Spain and Portugal during the winter of 1860-1861, las medidas que se toman tras el fallecimiento de un británico durante una de las oleadas de la epidemia del cólera que acecha a la ciudad, así como el desarrollo y evolución de la pandemia durante los meses de verano:

During the month of June 1860 cholera had visited Malaga, and prevailed to some extent for six weeks, and then for a time disappeared, until Sunday the 10th of August, when a heavy cloud and fog hung over the town during the day and succeeding night: most wonderful to relate, it was found next morning that there had been about eight hundred cases of cholera during the night; of these, forty-eight proved fatal the first day, and forty the second day; a few cases were afterwards fatal; and the malady was then said to have disappeared (Dunbar, 1862: 61-62).

Añade en su relato que las autoridades locales son muy susceptibles en este punto y, aunque Málaga se consideraba en la época el puerto más insalubre del Mediterráneo, se afanan en hacer ver que su puerto estaba libre de enfermedades infecciosas. Y pone el ejemplo de un caballero inglés que murió en Málaga durante su estancia, cuyo deceso el cónsul quiso participar al gobierno inglés que había sido provocado por el cólera, pero el gobernador malagueño no permitió que esto se hiciera, e insistió en que solo se telegrafiaría la información de «muerte repentina».

También se localizan noticias sobre la comunidad extranjera afincada en Málaga y sus condiciones sanitarias a través de los testimonios de viajeros españoles. Por medio de las cartas al director del periódico El Defensor de Granada: diario político independiente, se describe, por parte de dos damas que fueron invitadas a una ceremonia de enterramiento en el Cementerio Inglés, la noticia del fallecimiento de la dama inglesa Sophia Howart, ${ }^{2}$ cuyo proceso de inhumación fue realizado durante la noche del 23 de julio de 1881. En su descripción, las damas reconocen el respeto de la ceremonia, pero echan en falta lo sublime de los salmos de la liturgia católica:

2 Libro de inhumaciones del Cementerio Inglés de Málaga, AMM, 96-Volumen-159, registro nº 283, ff. 43v $-44 \mathrm{r}$. 
La escritura de la memoria sanitaria: el Libro de inhumaciones del cementerio inglés...

Una vez allí, encontramos el cadáver ante la capilla, en el suelo, y en medio del precioso jardín que forma el Cementerio. Dicho cadáver había sido conducido á aquel paraje la noche anterior, en un coche fúnebre y sin luces, pues su tradicional costumbre las prohíbe. Queda allí depositado en un nicho, durante la noche, y abierta la capilla, donde están los empleados y familiares, guardándolo religiosamente.

A la hora del entierro, y ya en el Cementerio todo el duelo, (en el que figuran los hijos y deudos más cercanos del finado), empieza el cura protestante sus rezos en inglés, revestido con sotana negra y estola del mismo color, y con una sobrepelliz particular, pues su forma es á modo de dalmática ó peínador blanco de señora, pronunciando todas las oraciones con ademan compujido y algo melodramático ante la fosa abierta, y derramando lágrimas, no sé si naturales ó de ordenanza, despues de los últimos responsos. Se coloca á seguda cuidadosamente el cadáver en la especie de nicho primorosamente fabricado en el suelo, y allí arrojan el cónsul de Inglaterra y los parientes más cercanos tierra y piedrecitas, que besan antes religiosamente. Más tarde se pone albahaca sobre el ataud, y un lúgubre silencio resta solo de esta ceremonia tristísima (...) (El defensor de Granada nº 297 - F.V., 1881: 3).

\section{EL LIBRO DE INHUMACIONES DEL CEMENTERIO INGLÉS DE MÁLAGA}

El Libro de inhumaciones del Cementerio Inglés de Málaga es una unidad documental que recoge toda la información relativa al enterramiento de cadáveres en este camposanto durante un periodo de 129 años. Entre todas las fuentes documentales directas generadas a lo largo del tiempo vinculadas al recinto sagrado, es esta -junto con la epigrafía funeraria inscrita en las lápidas (Marchant, 2005; RAmírez, 2016)- la única que nos brinda un conocimiento más amplio sobre la realidad social de los extranjeros afincados en Málaga, o que visitaron la ciudad, allí inhumados.

A día de hoy este libro no se encuentra en su emplazamiento original, puesto que la Fundación Cementerio Inglés de Málaga lo cedió por un plazo de veinte años al Ayuntamiento de la ciudad (prorrogable posteriormente por periodos de cinco años), para su custodia y conservación en el Archivo Municipal de Málaga. Este acuerdo fue firmado por el presidente de la Fundación Cementerio Inglés de Málaga, D. Bruce Alexander McIntyre y la Concejala de Cultura, Da . Gemma del Corral Parra, el 10 de octubre de 2014. ${ }^{3}$ La signatura que disfruta en su nuevo emplazamiento es AMM 99- Volumen- 159. Hasta entonces, había sido custodiado de forma ininterrumpida desde 1831 en el Consulado Británico de la ciudad. Los registros de decesos escritos en el Libro se inician en el año 1831, coincidiendo con la apertura e inauguración de este recinto sagrado, y finalizan en el año 1960, ya entrados en la segunda mitad del siglo xx. Como se ha explicado en la introducción, y por las razones argumentadas, nos centraremos en el estudio de los primeros 69 años, correspondientes al siglo XIX.

3 Convenio de Cesión de uso del Libro de Registro de las Inhumaciones del Cementerio Inglés de Málaga 1831-1860, suscrito entre el Excmo. Ayuntamiento de Málaga y la Fundación del Cementerio Inglés de Málaga (22-12-2014). Ayuntamiento de Málaga, Registro de Convenios y Protocolos, Doc. 489.420, $\mathrm{n}^{\circ}$ Orden 463 (Inscripción definitiva). 
Compuesto de un total de 99 folios, este libro presenta una estructura documental propia y concreta en cada uno de ellos, que es la siguiente tabla:

TABLA 1

Estructura de los folios del Libro de inhumaciones del Cementerio Inglés de Málaga

\begin{tabular}{|l|l|}
\hline \multicolumn{1}{|c|}{ Folio vuelto } & \multicolumn{1}{|c|}{ Folio recto (numerado) } \\
\hline Name & When buried \\
\hline Sex & Where Service scad by where buried \\
\hline Age & Signature of Informant \\
\hline Social condition & Signature of Consul as registrer \\
\hline Rauh* or occupation & Remarks \\
\hline When and where born & \\
\hline Place and time of Death & \\
\hline Cause of Death & \\
\hline
\end{tabular}

Elaboración propia.

Teniendo en cuenta el formato y distribución de la información en los libros de registro de defunción españoles de la época, se pueden apreciar notables diferencias (Brel, 1999; Carretero, 2009; Galende y Ávila, 2011). Tanto es así, que en el Libro de inhumaciones del Cementerio Inglés carecemos de información específica sobre la familia del difunto, o si ha realizado testamento. Elementos que aparecen en los registros españoles de defunción, junto con el hecho de recibir auxilio eclesiástico, que quizá se deban a la antigua tradición de demostrar el origen puro del linaje. Ejemplo de ello lo vemos reflejado en la copia de la partida de defunción de James Cameron, ${ }^{4}$ fallecido en Málaga a consecuencia de un tumor pútrido en la pierna, el 18 de septiembre de 1869. Se registra su fallecimiento en el Libro de Inhumaciones del Cementerio Inglés de Málaga, así como en los autóctonos de la ciudad. Este hombre sí testa pero no recibe ningún auxilio espiritual, y se indica que recibe sepultura en el cementerio «de los de su clase». ${ }^{5}$ Por ello, en el caso del Libro de inhumaciones del Cementerio Inglés de Málaga, la información albergada en el registro de defunción se corresponde principalmente con la del individuo inhumado, siendo la epigrafía el basamento para conocer los lazos familiares o de amistad del difunto (RAMíreZ, 2016: 84).

La información que se desprende de los registros de defunción del Libro de inhumaciones durante el siglo XIX ofrece los siguientes porcentajes de inhumados con relación a sexos y tramos de edad:

4 Libro de inhumaciones del Cementerio Inglés de Málaga, AMM 99-Volumen-159, registro nº 201, ff. 31v -32 r.

5 Libro de defunciones de 1869, mes de septiembre, fol. 6 (partida $\mathrm{n}^{\circ} 21$ ). 
GRÁFICO 1

Inhumados del Cementerio Inglés de Málaga (1831-1900)

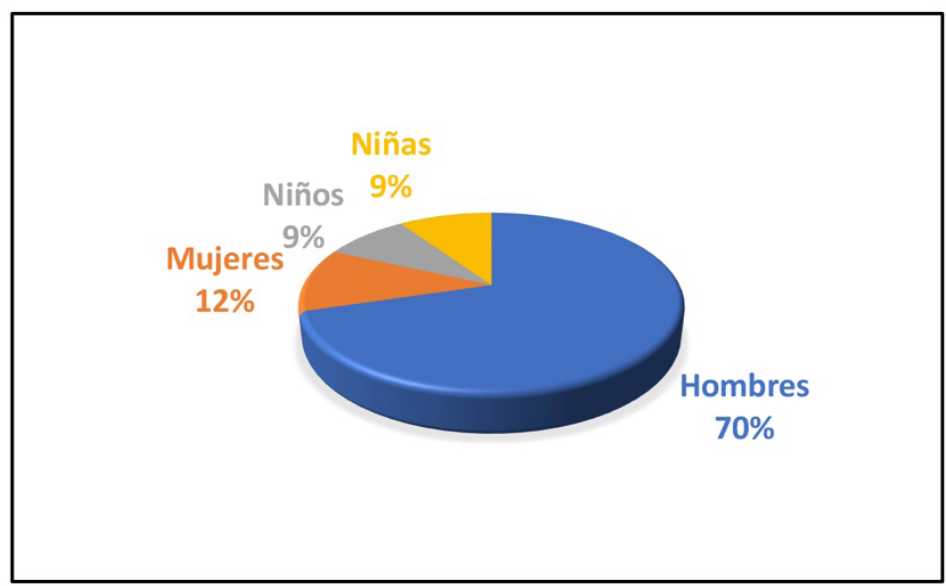

Elaboración propia.

Para el siglo XIX contamos con un total de 347 registros de decesos. De estos, debemos destacar el registro $\mathrm{n}^{\circ} 48$, que se encuentra vacío producido por un salto, así como dos registros que no aparecen incluidos en la numeración y que pertenecen a los inhumados por el naufragio en el Puerto de Málaga de la fragata Gneisenau, en diciembre de 1900 (MATEO, 2001: 163-176; PÁEz, 1980: 3-11). También incluimos el registro $n^{\circ} 335$, en el que solo aparece un nombre, sin añadir el resto de la información. De este total, se desprende que la mayoría de inhumados en el camposanto son hombres, seguidos del conjunto total de niños $\mathrm{y}$, por último, de mujeres. También se deslindan 53 registros en los cuales se desconoce la patología que ha causado la muerte del inhumado, así como otra información relevante sobre su origen o circunstancias.

Cada registro, además de su numeración y de los datos básicos del inhumado (nombre, edad, sexo, estado civil, condición y lugar de ocupación) incluye el lugar de defunción, la causa de muerte, el lugar de sepultura con su registro, así como otros datos relevantes. Cada registro irá acompañado de la verificación y firma de cada cónsul, quien generalmente es el autor del registro, siendo en esta centuria cinco los cónsules británicos en la ciudad. Estos son el Cónsul británico fundador William Mark, su hijo William Penrose Mark, el Cónsul R. Wikilson, el Cónsul Charles Toll Bidwel y el Cónsul Alexander Finn. También se incluye la firma del Vicecónsul John Atkins Mark y del Cónsul interino, John Damm.

En torno a la causa de muerte del inhumado, principal elemento de análisis en 
este estudio, hay que tomar en consideración el lugar donde se produce el deceso. Aunque en la mayoría de los registros el lugar de fallecimiento es Málaga capital, también se incluyen otros lugares de la geografía española como Barcelona, Granada y Almería. Y dentro de la provincia de Málaga destacan Benalmádena, Torremolinos, Alhaurín, el paraje natural de Bombicher o Carratraca. Entre los inhumados con vinculación directa al ámbito marítimo, destaca como lugar de fallecimiento el Puerto de Málaga (Malaga Harbour), el mar (on the see) o la embarcación a la que está vinculado. En los últimos registros finiseculares, sí se aprecia más información en relación al lugar físico concreto donde se produce el deceso. Se observa así un cambio cualitativo, señalando no solo el distrito donde se produce la muerte (como ocurría en la designación «Málaga Harbour» - Puerto Centro histórico de la ciudad -), sino la dirección postal exacta. Entre estas, y junto a referencias a calles del casco histórico, como San Juan o Alameda de los Tristes - actual Alameda de Colón - , abundan las calles aledañas al propio Cementerio Inglés de la ciudad, situado en las inmediaciones del Barrio de la Malagueta, como las calles Reding, Ibarra, Avenida de Príes, Paseo de Sancha o Monte Sancha, entre otros. También en ocasiones se alude a casas o villas determinadas donde moraron los inhumados, como serán «La Perla» o «Villa Maruja» (PGOU, 2010: 33). ${ }^{6}$ O incluso a entornos laborales como el grupo de casas de trabajadores textiles organizados junto a la fábrica Industria Malagueña, fundada en 1846 por las familias Heredia y Larios y disuelta en 1905 (JIMÉnEZ QuINTERO, 2009: 115-124). En cuanto a aquellos enfermos que fallecen en un establecimiento hospitalario, la única referencia encontrada es al Hospital Noble de Málaga, situado a muy pocos metros del Cementerio Inglés (MARChANT y BARRENA, e.p.).

Estas referencias nos dan la certeza de la pervivencia y presencia de los extranjeros en la ciudad que acaban asentándose en la misma. Aunque en el resto de los registros de decesos no aparecen alusiones precisas al lugar donde fallecen los extranjeros que se encuentran de paso en la ciudad, se puede completar esta información a través de los registros de defunción que se localizan en la página web Family Search, que corroboran que a muchos de ellos los sorprenderá la muerte en los establecimientos hoteleros de la ciudad. El Dr. More (1874: 116-117) hace una descripción de estos establecimientos:

The principal hotels in Malaga are the Alameda, Victoria, and Oriente, all situated on the Alameda. The two first are chiefly fequented by English and the latter by French travellers but not exclusively; the usual charge in al lis from 25 to 35 reals, about five to seven shilling, a day; this includes lodging, breakfast, dinner, and wine. It is esencial to invalids to choose rooms facing the Alameda, as those which have not a southern aspecto are quite unfit for them, there being a difference of from 6 to $10 \mathrm{deg}$. in the day temperatura between apartments on the north and south side of the house, and a still greater variation at night.

Añade que estos hoteles están adornados de forma modesta, porque hay

6 Villa Maruja es un edificio protegido, de grado Arquitectónico II, situado en el Paseo del Limonar, $\mathrm{n}^{\circ}$ 41. Plan de Ordenación General Urbanística (PGOU) -Zona A- Limonar-Malagueta. 2010 [en línea]. Disponible en: http:// www.pgou.eu/Malaga.htm [Consulta: 04/05/2020] 
que tener en cuenta que en el Sur de España muchos objetos que los ingleses contemplan como indispensables en el hogar (cortinas, alfombras, ...) serían un inconveniente por los parásitos que se desarrollan en zona portuaria y evitarían la circulación libre del aire, tan necesaria en un clima cálido. Añade que las familias españolas creen, por lo general, en el carácter contagioso de la tisis y no quieren alojar a invalids en sus casas, por ello recomienda la pensión de la Sra. Castiglione. Salva también las casas del muelle, que están bien situadas, pues ninguna de las casas de pupilos o pensiones son convenientes para los que tienen salud débil, porque están generalmente mal situadas, las habitaciones son pequeñas y mal amuebladas, la cocina horrible y solo se habla andaluz, al que Madden considera una forma desvirtuada del español.

Otra de las consideraciones a tener en cuenta son las patologías que se manifiestan en la ciudad de Málaga durante esta centuria. Atendiendo a las enfermedades de carácter epidémico, serán los brotes de fiebre amarilla los que asolen a buena parte de la población durante las primeras décadas del siglo XIX, seguido de los brotes de cólera morbo (DíAz, 1903: 557-584; CARrILlo-GARCía, 1980; Delange, 2003). Al mismo tiempo, no podemos obviar patologías de carácter infeccioso como tifus, difteria, tisis, consunción o viruela, entre otros, que se desarrollan no solo en la capital malacitana sino también en todo el territorio nacional y que, por tanto, constatan también su aparición o desarrollo dentro del Libro de Defunciones (DE LA FIGUERA, 2009: 151-171).

\subsection{El registro de los decesos de infantes}

Los infantes constituyen el 18\% de los inhumados en el camposanto durante la centuria decimonónica. Este dato elevado se corresponde con el alto índice de natalidad unido a la elevada mortalidad que caracteriza esta época. En la mayoría de las ocasiones, y como podemos apreciar en la tabla 2, estos niños no alcanzaban los cinco años de edad en el momento de su muerte. Es significativo que muchos de ellos murieran antes del año; otros, sin llegar a sobrepasar el día (ROBLES, 2002).

A diferencia de los hombres y las mujeres, y por razones obvias, la información relativa a la condición social o a la ocupación profesional, aparece en la mayoría de los casos en blanco o con el siguiente símbolo «------». Salvo en excepciones, donde en la primera clasificación de las mencionadas, aparece información relativa al progenitor o se especifica infant. También aparecen los términos boy y girl referentes al sexo de los inhumados, aunque es más frecuente el uso de male y female para tal caso.

La mayor parte de estos niños inhumados son malagueños e hijos de los extranjeros afincados en la ciudad, por lo que son muy pocos los pequeños de origen extranjero. Entre estos últimos, destacan lugares de nacimiento reseñados 
como Gran Bretaña (Liverpool, ${ }^{7}$ Exeter ${ }^{8}$ Edimburgo, ${ }^{9}$ ), Alemania (Hannover), ${ }^{10}$ EEUU (Boothbay Harbour), ${ }^{11}$ Italia (Toscana) ${ }^{12}$ y Rusia. ${ }^{13}$

Los primeros inhumados fueron M. Elisabeth Andrew, de solo 19 meses de edad y Franco Brown, de 16 meses de edad. ${ }^{14}$ En estos primeros casos no se registra la patología que ha constituido la causa de muerte. Previamente a estas dos inhumaciones de infantes, localizamos el registro $n^{\circ} 9$, en el que apenas consta la fecha de nacimiento y defunción, producida en el mismo día, y que hace referencia a dos niños. ${ }^{15}$

Centrándonos en las causas de muerte, tanto en niños como en niñas, se constata que se produce un mayor número de fallecimientos en los intervalos de 0-5 años, que en el intervalo de 5-10 de edad. Entre las patologías reseñadas como causa de muerte destacan en general aquellas relacionadas con el aparato digestivo, como la diarrea, el $\operatorname{crup}^{16}$ y la dentición. Pero también se alude a enfermedades de carácter infeccioso como la tisis o el cólera (DE LA FIGUERA, 2009: 156-159), esta última causa tristemente afamada tras las varias oleadas epidémicas

7 Libro de inhumaciones del Cementerio Inglés de Málaga, AMM 99-Volumen-159. Registro n ${ }^{\circ} 12$, ff. 4v - 5r. M. Elizabeth Andrew - Female (1 year and 7 months) (Liverpool. May, 11. 1835 - Malaga. December, 19. 1836).

8 Libro de inhumaciones del Cementerio Inglés de Málaga, AMM 99-Volumen-159. Registro nº 179, ff. 28v$29 \mathrm{r}$.

Charles Uctermarek Hardy - Male (8 months) (Exeter. February, 2. 1864 - Malaga. October, 15. 1864) (Diarrhea).

9 Libro de inhumaciones del Cementerio Inglés de Málaga, AMM 99-Volumen-159. Registro no 63, ff. 11v -12 r.

Catherine Charlotte Anne Eliza Boyle - Female (2 years) (Edinburgh. February, 9. 1849 - Malaga. December, 17. 1851) (Gastric fever). Registro $n^{\circ}$ 65, ff. 12v -13r. Greenne Hepbourn Boyle - Male (3 years) (Edinburgh. February, 8. 1848 - Malaga. January, 28. 1852) (Croup). Registro n ${ }^{\circ} 209$, ff. 32v - 33r. Louise Charles Hamilton Massey Verschoyle - Male (23 months) (Edinburgh. November, 29. 1868 Malaga. November, 28. 1870) (Brain fever).

10 Libro de inhumaciones del Cementerio Inglés de Málaga, AMM 99-Volumen-159. Registro $\mathrm{n}^{\circ} 53, \mathrm{ff} .10 \mathrm{v}$ $-11 r$.

Jhnke Taaks - Male (2 years) (Bensersiel, Hanover. September, 30. 1847 - Malaga. October, 4. 1849) (Teething Fever).

11 Libro de inhumaciones del Cementerio Inglés de Málaga, AMM 99-Volumen-159. Registro $\mathrm{n}^{\circ}$ 107, ff. $18 \mathrm{v}-19 \mathrm{r}$.

Edward Sewall Wylie - Male (one year) (Boothbay harbour, U.S.A. February, 3. 1855 - Malaga. September, 14. 1856) (Dysentery).

12 Libro de inhumaciones del Cementerio Inglés de Málaga, AMM 99-Volumen-159. Registro $\mathrm{n}^{\circ}$ 175, ff. $27 \mathrm{v}-28 \mathrm{r}$.

Beatrice Treshella - Female (9 years) (Peteccio nen Pistoia, Tuscany. February, 8. 1855 - Bombicher, May, 6. 1864) (Disease of the heart).

13 Libro de inhumaciones del Cementerio Inglés de Málaga, AMM 99-Volumen-159. Registro nº 333, ff. $50 \mathrm{v}-51 \mathrm{r}$.

Paul Burger - Male (7 years) (Rusian Subject - Malaga. September, 20. 1894).

14 Libro de inhumaciones del Cementerio Inglés de Málaga, AMM, 96-Volumen-159, registro $\mathrm{n}^{\mathrm{o}} 12$, ff. 4v 5r.; registro $\mathrm{n}^{\circ} 25$, ff. $6 \mathrm{v}-7 \mathrm{r}$.

15 Libro de inhumaciones del Cementerio Inglés de Málaga, AMM, 96-Volumen-159, registro n 9, ff. 4v - 5r. 16 Conocida también como difteria o laringotraqueobronquitis. «Enfermedad específica, infecciosa y contagiosa, caracterizada por la formación de falsas membranas en las mucosas, comúnmente de la garganta, en la piel desnuda de epidermis y en toda suerte de heridas al descubierto, con síntomas generales de fiebre y postración». Real Academia Española, Diccionario de la lengua española [en línea]. Disponible en: https:/ / dle.rae.es/difteria\#5yq3Cxf [Consulta: 08/05/2020]. 
que atravesó Málaga durante el siglo xix (Delange, 2003; Albuera, 1998: 53-56).

En el intervalo de 5-10 años, siendo el minoritario, destacan los casos de Beatrice Treeshella, que fallece a los 9 años en 1864 de una afección al corazón ${ }^{17}$ o el de Adolphus Royanner, nacido en Linares (Jaén), que fallece a los 10 años en 1869 como consecuencia de una enfermedad de la vejiga. ${ }^{18}$

Muchos de estos niños mantienen o participan de vínculos familiares y esa relación no solo la podemos comprobar por el apellido, sino en el apartado Where de la hoja de registro de inhumación, que hace referencia a la localización de la lápida, y en Remarks, donde se puede hacer alusión a otros datos familiares significativos.

Así, como ejemplo, de la familia del Cónsul Británico William Mark, localizamos en los registros del Libro de inhumaciones a sus nietos William Charles Mark, que fallece en 1855 y del que desconocemos la causa de su muerte, y Reginald Penrose Mark, que fallece en 1862 a los tres meses de edad, a consecuencia de unas convulsiones. Ambos son hijos del cónsul británico William Penrose Mark y de su esposa, Helen Elizabeth Mark. ${ }^{19}$

También destacamos a Joseph Mark, de la misma edad, que fallece en 1875 de marasmo o lo que es lo mismo, enflaquecimiento del cuerpo. ${ }^{20}$ Es hijo de John Atkins Mark, Vicecónsul Británico y de su esposa, Elizabeth Josephine Mark. Dichos infantes fallecen en la residencia familiar, a caballo entre Málaga capital y Benalmádena, y sus restos reposan en el panteón familiar ${ }^{21}$ (grave $n^{0}$ 50/220, section G) (MARCHANT, 2005: 66-73).

Los registros 256 y 257 corresponden a la familia Bjerre, donde ambos hermanos fallecen en el mismo año, de meningitis. La niña, Jacobine Petrine, a los 23 días, y el niño, Svena Emil Winslas, a los 3 años. Por otro lado, la familia Reuss Christine pierde en el intervalo de 3 meses a sus dos pequeñas, Sophie Amelia, de 4 meses, que fallece a consecuencia de una pleuroneumonía, y Josephine Elizabeth, con 2 años y 5 meses, que fallece a causa del crup.

Vinculados al entorno de la industria malagueña decimonónica, encontramos a las hermanas Victoria y Margaret Crawshaw, de tres y dos años y medio respectivamente. En el registro 334, correspondiente a Margaret, se refiere que fallece a consecuencia de fiebres en la Fábrica Industrial en 1894, además de indicar en la misma partida que el padre es ingeniero. También en su Remarks, aparece la siguiente frase In her sister's grave, $n^{\circ} 311$, por lo que sabemos que ambas

17 Libro de inhumaciones del Cementerio Inglés de Málaga, AMM, 96-Volumen-159, registro no 175 , ff. 27v $-28 \mathrm{r}$.

18 Libro de inhumaciones del Cementerio Inglés de Málaga, AMM, 96-Volumen-159, registro $\mathrm{n}^{\circ}$ 200, ff. $31 \mathrm{v}-32 \mathrm{r}$.

19 Libro de inhumaciones del Cementerio Inglés de Málaga, AMM, 96-Volumen-159, registro no 94, ff. 16v $-17 \mathrm{r}$.; registro $\mathrm{n}^{\mathrm{o}} 161$, ff. $25 \mathrm{v}-26 \mathrm{r}$.

20 Real Academia Española, Diccionario de la lengua española [en línea]. Disponible en: https://dle.rae. es/marasmo?m=form [Consulta: 08/05/2020].

21 Billion Graves (Joseph Mark) [en línea]. Disponible en: https://billiongraves.es/grave/JosephMark/25382334 [Consulta: 08/05/2020]; (Reginald Penrose Mark) [en línea]. Disponible en: https:// es.findagrave.com/memorial/128778884 [Consulta: 08/05/2020]; (William Charles Mark) [en línea]. Disponible en: https:/ / es.findagrave.com/memorial/128778885 [Última consulta: 08/05/2020]. 
hermanas descansan juntas en la misma sepultura (grave $n^{0} 311 / 334$, section $\left.E\right) .{ }^{22}$

\subsection{Los decesos femeninos}

Las mujeres son el grupo minoritario entre los inhumados del camposanto para estas fechas, representando el $12 \%$ del total de inhumados. Este porcentaje viene derivado de que la mayoría de los varones extranjeros que se encuentran en Málaga durante esta etapa lo hacen motivados por negocios, en su mayoría vinculados al ámbito comercial, dándose la circunstancia de que, en un primer momento, sus esposas podían permanecer en su ciudad de origen.

En cuanto a su estado civil, se localizan esposas, viudas y solteras. En cuanto a su profesión, la mayoría de ellas se dedican al ámbito doméstico, en empleos o tareas como cuidadora de niños, cocinera o costurera, entre otros (MARCHANT, 2004: 161-165). En el seno de la información proporcionada por el registro de la inhumación, existen lagunas sobre la referencia de ocupación profesional de algunas mujeres enterradas en el camposanto. De hecho, así lo evidencian varios registros donde, en el espacio destinado a referir la profesión u ocupación de estas mujeres, aparece su filiación marital. En otros casos, como ocurre con la viuda del Cónsul fundador, ${ }^{23}$ Emma Bedweld, nacida en Londres el 1 de enero de 1788 y fallecida en Málaga el 22 de noviembre de 1859, se necesita del apoyo epigráfico para poder conocer algunos datos más acerca de su vida y su vinculación a la tierra donde reposa (MARCHANT, 2005: 70-71).

Atendiendo a las causas de muerte de estas mujeres, se puede ver que el rango de edad en el que se producen los decesos es a partir de los 20 años, como se aprecia en la tabla $n^{\circ} 3$. En las edades más tempranas se desarrollan con mayor profusión las enfermedades y dolencias cardiacas, así como todo tipo de afecciones respiratorias, entre ellas la tisis y la pleuresía. Edwin LEE (1854: 80-90) determina para este hecho que el clima malagueño era perjudicial para las enfermedades cardiacas, mientras que sí destacaba el beneficio para las enfermedades respiratorias. La primera mujer sepultada será Julie Sandeman, el 20 de febrero de 1845, a consecuencia de la tisis, que le arrebata la vida con tan solo 29 años. ${ }^{24}$

A diferencia de los niños y de los hombres, que analizaremos en las páginas siguientes, encontramos en las mujeres causas de muerte registradas como el cáncer y la leucemia, en concreto en los casos de Henrrietta Elizabeth Hellman, de 31 años y procedente de Hamburgo, que fallece en nuestra ciudad en 1876 de leucemia. ${ }^{25} \mathrm{O}$ el cáncer que acabó con la vida de Mary Heaton en 1884, a la edad

22 Libro de inhumaciones del Cementerio Inglés de Málaga, AMM, 96-Volumen-159, registro no 279, ff. 42v$43 \mathrm{r}$.; registro $\mathrm{n}^{\mathrm{o}} 281$, ff. $43 \mathrm{v}-44 \mathrm{r}$.

23 Libro de inhumaciones del Cementerio Inglés de Málaga, AMM, 96-Volumen-159, registro n 138, ff. $22 \mathrm{v}-23 \mathrm{r}$.

24 Libro de inhumaciones del Cementerio Inglés de Málaga, AMM, 96-Volumen-159, registro nº41, ff. 8v-9r. 25 Libro de inhumaciones del Cementerio Inglés de Málaga, AMM, 96-Volumen-159, registro n²53, ff. $39 \mathrm{v}-40 \mathrm{r}$. 
La escritura de la memoria sanitaria: el Libro de inhumaciones del cementerio inglés...

de 48 años. $^{26}$

Otras de las enfermedades que durante siglos han causado la mortalidad femenina han sido aquellas provocadas por el hecho de dar a luz. Como será el caso de Elizabeth Williams, mujer de David Williams, que fallece de parto prematuro a bordo de la embarcación Margaret en aguas de Torremolinos, en 1857, a la edad de 38 años. ${ }^{27}$ O Harriet Amelia Hardy, que con 33 años pierde la vida a principios de 1867, tras complicarse el parto y producirse una peritonitis puerperal (BAudeloceue, 1831). O la mujer del Vicecónsul Británico John Atkins Mark, Elizabeth Josephine Mark, que fallece en 1875 a los 40 años, tras dar a luz a su hijo, Joseph Mark, quien también perdería la vida tres meses después. ${ }^{28}$

\subsection{Los decesos masculinos}

El último de los grupos que sometemos a análisis, no por ello el menos importante, es el de los hombres. Este representa el 70\% de las inhumaciones llevadas a cabo durante el siglo xIx en el cementerio inglés de Málaga (Tabla 4). La mayor parte de ellos son comerciantes y gente vinculada al mar, pero atendiendo a tipos de profesión y clase social, se connota en muchos casos que se trata de gente adinerada y culta, destacando cónsules y vicecónsules de varios países, oficiales militares, sacerdotes y jueces, entre otros.

El primer inhumado, varón adulto, del camposanto fue el capitán del buque británico Cicero, George Stephens, ${ }^{29}$ que fallece por ahogamiento en el mismo puerto de Málaga el 20 de enero de 1831. La leyenda urbana apunta que la causa de muerte estuvo provocada por una intoxicación etílica previa (HerEDIA y Aguilera, 2019: 25). No obstante, la figura del primer inhumado en este nuevo recinto siempre permaneció eclipsada y a la sombra del irlandés Robert Boyd. Tanto en los distintos testimonios recogidos en la literatura de viajes, incluso en los estudios más recientes vinculados al estudio patrimonial de este cementerio, se indica que el de Robert Boyd fue el primer enterramiento que se llevó a cabo en este recinto en $1831 .{ }^{30}$ Robert Boyd sería fusilado en las playas de San Andrés el 11 de diciembre de 1831, junto al General Torrijos y el resto de sus hombres, tras luchar contra el absolutismo del monarca Fernando VII (GuILLÉN, 1983: 654-657), y

26 Libro de inhumaciones del Cementerio Inglés de Málaga, AMM, 96-Volumen-159, registro n³01, ff. $45 \mathrm{v}-46 \mathrm{r}$.

27 Libro de inhumaciones del Cementerio Inglés de Málaga, AMM, 96-Volumen-159, registro n¹13, ff.19v$20 \mathrm{r}$.

28 Libro de inhumaciones del Cementerio Inglés de Málaga, AMM, 96-Volumen-159, registro n²45, ff. $37 \mathrm{v}-38 \mathrm{r}$.

29 Libro de inhumaciones del Cementerio Inglés de Málaga, AMM, 96-Volumen-159, registro n ${ }^{\circ} 1$, ff. 3v - 4r. 30 En torno a la literatura de viajes se aglutinan las diversas descripciones que se realizan en torno a la muerte de Robert Boyd. De hecho, es el mismo Richard Ford (1845) en una de sus correspondencias, el que bromea e ironiza sobre la ilusión del Cónsul Británico de estrenar el camposanto, en este caso con la esposa del propio Ford, que presenta una débil salud. En las líneas siguientes de su misiva, redactada desde Sevilla, describe cómo el cónsul reclama el cuerpo de Boyd para su inhumación (PROTHERO, 1905: 75). También en estudios recientes vinculados al ámbito patrimonial de los cementerios, se alude a que el mismo Boyd es el primer enterrado en el cementerio protestante (TARRÉS y MORERAS, 2012: 271). 
recibiría sepultura en el Cementerio Inglés de Málaga.

A partir del año 1832, empiezan a enterrarse en el cementerio extranjeros de otras nacionalidades diferentes a la británica. El primero de ellos será el Cónsul sueco John Bolin, cuyo apellido daría origen a una de las grandes familias malagueñas del momento (Heredia y Aguilera, 2019: 11-12). La importancia de realizar enterramientos de ciudadanos de otras naciones queda plasmada en la obra estadounidense The American and foreign Christian Union, donde se recoge la escritura última de las lápidas de Thomas Clerke y de Ethelred F. Coddington, ambos fallecidos de tisis (Figura 1). También se agradece la empresa llevada a cabo por el cónsul británico William Mark, al no permitir que los súbditos americanos acaben siendo abandonados a su suerte en las playas malagueñas, sin un final digno (VV.AA., 1859: 97-99).

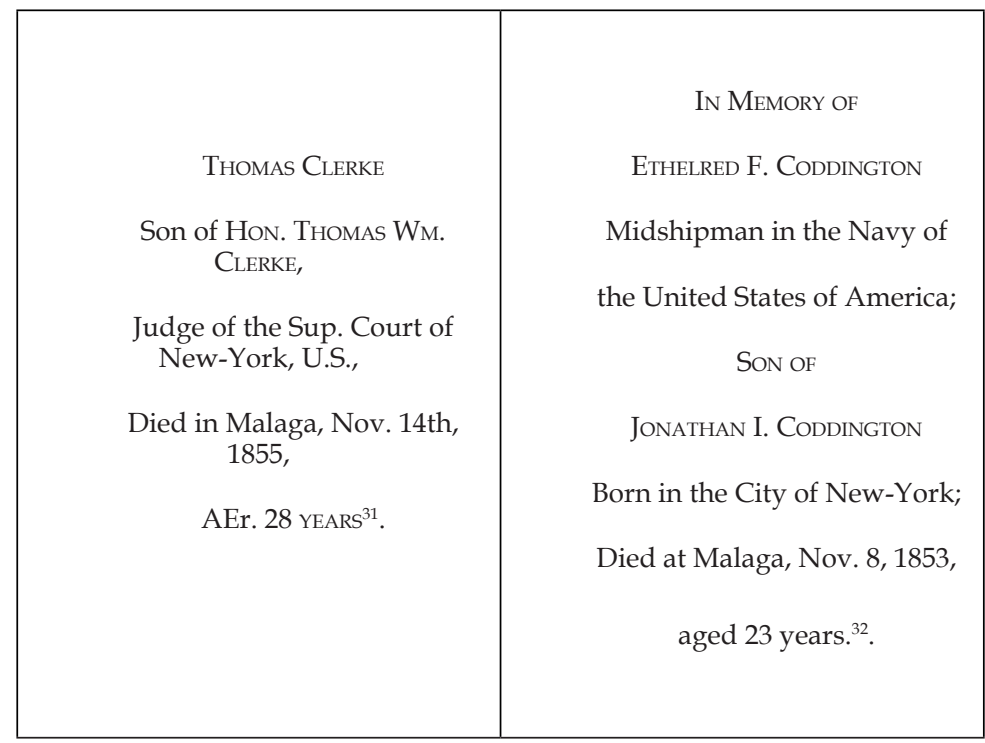

Figura 1. Lápidas de Thomas Clerke y Ethelred F. Coddington. Elaboración propia.

Si atendemos a la causa de muerte provocada por enfermedades de carácter epidémico, debemos focalizar nuestra atención en las diversas epidemias de cólera que atraviesa Málaga durante el siglo xIX. Como apunta Josef Mendoza, desde centurias anteriores se conocía la presencia del cólera morbo en la ciudad, pero será para el año 1831 cuando encontremos sus síntomas entre la población, aunque con poca virulencia en el número de víctimas (CARRILlo y MENDOZA, 1834).

31 Find a Grave (Thomas Clerke) [en línea]. Disponible en: https://es.findagrave.com/memorial/197795462/thomas-clerke [Consulta: 08/05/2020]

32 Find a Grave (Ethelred F. Coddington) [en línea]. Disponible en: https://es.findagrave.com/memorial/192436567 [Consulta: 08/05/2020] 
Para el otoño de 1833, la epidemia apareció con mayor fuerza y es aquí donde se incardinan los casos del sirviente Thomas Gilbert o del obrero industrial John Bromley. Esta epidemia no volverá hasta el año 1854, cuando se cobre la vida de Morgan Davies, marinero de la goleta Grasshoper, y en el verano del año siguiente, donde destacan los decesos del comerciante Thomas Gordon y el capitán Edward John Mauger, quien falleció a bordo de la embarcación Lady Mausele (Heredia y Aguilera, 2019: 12). Pero quizás la muerte más conocida y recogida en distintos testimonios hebdomadarios del momento será la del médico Joseph Noble, ${ }^{33}$ fundador del Hospital Noble de la ciudad, que fallece en Málaga el 6 de enero de 1861, tras atender a un enfermo infectado de cólera que se encontraba en la Fonda de la Alameda (LARA, 2005: 189).

En relación con las enfermedades del aparato respiratorio destaca la tisis (consumption), que tanto se desarrolla en la época en la ciudad mediterránea. Buena prueba de ello será el gran número de inhumados con esta dolencia como causa de fallecimiento. Es necesario también tener en cuenta que, al ser Málaga una ciudad de clima muy benigno, se recomienda su visita para solventar las dolencias pulmonares, por lo que encontramos muchos casos de inhumados con enfermedades vinculadas al aparato respiratorio (LEE, 1854: 80). Entre ellos destacamos los miembros varones de la familia Mark, la familia del Cónsul fundador, que fallecen por afecciones de carácter respiratorio. El propio Cónsul fundador, por bronquitis, el 13 de enero de 1849; y sus hijos William Penrose Mark, de fiebre tifoidea, el 20 de enero de 1872, y John Atkins Mark, de bronquitis crónica, el 18 de febrero de 1881.

Otras de las causas de muerte que destacan entre los inhumados varones son aquellas que clasificamos en el bloque de «Accidentes, suicidios y muertes violentas» en la Tabla $n^{\circ} 4$. Como señala Albuera (1998: 135):

En una sociedad como la malagueña, tan polarizada desde el punto de vista económico y sumida en una profunda crisis, la criminalidad ocasional alcanza niveles muy importantes. A los delitos contra la propiedad que culminan en derramamiento de sangre, habría que añadir dos factores aún presentes a finales de siglos: el código de honor y la venganza personal o familiar.

El mismo autor hace alusión en la obra a los suicidios en la ciudad de Málaga. Para principios del siglo xx, en concreto el año 1903, se indica que el número de suicidios en la ciudad llega a ser incluso superior al número de muertes violentas (Albuera, 1998: 339-340).

Estas causas de muerte, en la mayoría de los casos, están vinculadas a la gente de la mar. Entre ellas se sitúa la del marinero John Scott, que fallece a causa de un accidente el 6 de septiembre de 1841; John Robertsen, escocés que fallece tras caer de uno de los palos del barco; o incluso la muerte provocada por la explosión de gas en la carga del carbón del bergantín Maria Brockelmann, como

33 Libro de inhumaciones del Cementerio Inglés de Málaga, AMM, 96-Volumen-159, registro $\mathrm{n}^{\circ} 151$, ff. $24 \mathrm{v}-25 \mathrm{r}$. 
le sucedería al alemán Johann Wittenburg ${ }^{34}$ en septiembre de 1856. Se localiza el caso de un supuesto suicidio, del británico Louis Schimidt, en diciembre de 1836, así como algunos casos de homicidio, algo bastante frecuente en la época y en el entorno. Entre estos últimos, la muerte del tripulante del bergantín inglés Dasher, Henry Hutting, ${ }^{35}$ que cae herido tras recibir una puñalada por la espalda en la Acera de la Marina, el 2 de septiembre de 1855; o el caso del ingeniero Thomas Hitchell, ${ }^{36}$ asesinado el 11 de diciembre de 1881, tras el disparo de un centinela español. No en vano ya por esa etapa Málaga era contemplada como ciudad de las tabernas, lugares donde se perdía la salud y se pervertía la moral, ocasionando así el aumento de la criminalidad:

La gente acomodada bebe poco: la gente pobre pocas veces pone el vino en su mesa; pero en cambio va á alguna de las ciento cincuenta y cinco tabernas que hay (á tal esceso monta su número) y allí espenden el jornal de su trabajo; allí gastan su salud, y pervierten su moral. Si en alguna parte hay necesidad de una sociedad de la templanza es en Málaga, y con ella, y con sus progresos mucho ganarían las costumbres, se disminuirían los crímenes, y también rebajaría el número de enfermedades entre esta clase abyecta y desgraciada (MARTínez, 1852: 312).

\section{CONCLUSIONES}

El presente estudio ha procurado, por un lado, rescatar un nuevo enfoque de estudio y análisis sobre las fuentes documentales relativas al Cementerio Inglés de la ciudad de Málaga; ya que, como se ha indicado, diversos trabajos de investigación llevados a término hasta el momento han prestado atención a la historiografía del recinto, sus valores artísticos y epigráficos o su reconocimiento como monumento en el conjunto histórico de la ciudad. La puesta en valor de una unidad documental, el Libro de inhumaciones, que hoy reposa entre el grueso de documentación que custodia el Archivo Municipal de la ciudad, nos ha permitido realizar un ejercicio de análisis diplomático en el que el registro escrito, la fórmula, entreabre la puerta a la realidad histórico-social.

La acotación temporal de la realidad documental a la segunda mitad del siglo XIX ha resultado fructífera, por la cohesión social encarnada tanto por los foráneos afincados en Málaga como por los invalids que visitaban la ciudad para restablecerse de su enfermedad. Lo cual ha facilitado el análisis al correr este paralelo, en un principio, al empoderamiento industrial de la ciudad de Málaga y, finalmente, a su debacle industrial. A partir de 1865, La Guerra de Secesión de los Estados Unidos ejercería un efecto negativo sobre la industria textil malagueña, iniciándose ese año un período de crisis económica en la provincia, que se

34 Libro de inhumaciones del Cementerio Inglés de Málaga, AMM, 96-Volumen-159, registro n¹08, ff. $18 \mathrm{v}-19 \mathrm{r}$.

35 Libro de inhumaciones del Cementerio Inglés de Málaga, AMM, 96-Volumen-159, registro no 99, ff. 17v -18 r.

36 Libro de inhumaciones del Cementerio Inglés de Málaga, AMM, 96-Volumen-159, registro nº 284, ff. 43v $-44 \mathrm{r}$. 
acentuaría en 1879 con la invasión de la filoxera, hasta desembocar, aderezado con las epidemias, en un lento proceso de deterioro industrial finisecular.

Los datos estadísticos elaborados a partir de la información contenida en cada área del registro documental de los decesos permiten elaborar una radiografía de una Málaga portuaria, internacional, cuya vida civil se organizaba interiorizando esta miscelánea: marineros extranjeros, fundamentalmente ingleses, comerciantes, trabajadores foráneos en las fundiciones autóctonas e invalids que visitaban el puerto mediterráneo para restablecer su salud encontrando en muchas ocasiones en él su morada de descanso eterno. Infantes sacudidos por el estertor de las pandemias y epidemias, escasas mujeres, vinculadas por lo general profesionalmente al cuidado de los menores, y hombres afectados por patologías hoy día prácticamente desaparecidas, o envueltos en parámetros accidentales y de criminalidad. Todos ellos perpetúan su memoria a través de la escritura, no última en esta ocasión, proferida e inscrita en piedra por los más íntimos y allegados al inhumado, sino emanada de la puma de un cónsul que, como máximo responsable del colectivo extranjero que representa en la ciudad, se afana por plasmar la dignidad de un enterramiento, antaño negado, construyendo, al paralelo, una historia de la salubridad en una ciudad española en el pleno apogeo histórico, más tarde debacle, de su desarrollo industrial.

Por otro lado, cabe señalar que el conocimiento de las patologías manifestadas que fueron causa de muerte durante la segunda mitad del siglo xIX por estos extranjeros, asentados o visitantes de la ciudad, y en un contexto tan concreto como el cementerio inglés de la ciudad de Málaga permite vislumbrar el avance médico-sanitario de la capital malacitana, así como entender cuáles eran las causas que hacían enfermar a sus vecinos o visitantes.

Con esta aproximación se ha pretendido también reivindicar un primer paso hacia la descripción profunda de la documentación adyacente a la de carácter sanitario conservada en nuestros archivos, locales y nacionales, para la etapa del siglo XIX español. Poniendo como ejemplo este Libro de inhumaciones del cementerio inglés de la ciudad de Málaga, hasta el 2014 custodiado por la autoridad consular y hoy día integrado en el sistema archivístico nacional. 


\section{ANEXOS}

TABLA 2

Mortalidad infantil en el Cementerio Inglés (1831-1900)

\begin{tabular}{|c|c|c|c|c|c|c|}
\hline \multicolumn{7}{|c|}{ Mortalidad infantil en el Cementerio Inglés (1831-1900) } \\
\hline \multirow[t]{3}{*}{ Causas de las defunciones } & \multicolumn{6}{|c|}{ Detalles de las defunciones por causas y edades } \\
\hline & \multicolumn{3}{|c|}{ Niños } & \multicolumn{3}{|c|}{ Niñas } \\
\hline & $0-1$ & $1-5$ & $5-10$ & $0-1$ & $1-5$ & $5-10$ \\
\hline Asthma (Asma) & & 1 & & & & \\
\hline $\begin{array}{l}\text { Atelectasis of the lungs (Atelectasia } \\
\text { de los pulmones) }\end{array}$ & 1 & & & & & \\
\hline Brain fever (Encefalitis) & & 1 & & 1 & 1 & \\
\hline $\begin{array}{l}\text { Bronchial obstruction (Obstrucción } \\
\text { bronquial) }\end{array}$ & 1 & & & & & \\
\hline Catarrh (Catarro) & & 1 & & & & \\
\hline $\begin{array}{l}\text { Cholera morbus } \\
\text { (Cólera Morbo) }\end{array}$ & & 1 & & & & \\
\hline Cholera (Cólera) & & 1 & & & & \\
\hline $\begin{array}{l}\text { Congestion cerebral (Congestión } \\
\text { cerebral) }\end{array}$ & 1 & & & & & \\
\hline Consumption (Tisis) & & & & 1 & 1 & \\
\hline Convulsions (Convulsiones) & 1 & & & 1 & & \\
\hline Croup (Crup - Difteria) & 1 & 3 & & & 1 & \\
\hline Diarrhea (Cronic) (Diarrea) & 1 & & & & 2 & \\
\hline $\begin{array}{l}\text { Disease of the Bladder (Enfermedad } \\
\text { de la vejiga) }\end{array}$ & & & 1 & & & \\
\hline $\begin{array}{l}\text { Disease of the heart (Enfermedad } \\
\text { del corazón) }\end{array}$ & & & & & & 1 \\
\hline Dropsy (Hidropesía) & 1 & 1 & & & & \\
\hline \multicolumn{7}{|l|}{ Dysentery (Disentería) } \\
\hline Enteritis (Enteritis) & & & & 1 & & \\
\hline Epilepsy (Epilepsia) & 1 & & & & & \\
\hline Fever (Fiebre) & & & & & 1 & \\
\hline Gangrenum Erisipela (Erisipela) & & & 1 & & & \\
\hline Gastric fever (Fiebre gástrica) & 1 & & & & 1 & 1 \\
\hline Gastroenteritis (Gastroenteritis) & & & & & 1 & \\
\hline $\begin{array}{l}\text { Inflamation of the brain } \\
\text { (Inflamación del cerebro - } \\
\text { Encefalitis) }\end{array}$ & & & & & 1 & \\
\hline
\end{tabular}


La escritura de la memoria sanitaria: el Libro de inhumaciones del cementerio inglés...

\begin{tabular}{|l|c|c|c|c|c|c|}
\hline $\begin{array}{l}\text { Inflamation of the bowels } \\
\text { (Inflamación de intestinos) }\end{array}$ & & & & & 1 & \\
\hline $\begin{array}{l}\text { Inflamation of the lungs } \\
\text { (Inflamación pulmonar) }\end{array}$ & & & & 1 & & \\
\hline Measles (Sarampión) & 1 & & & & & \\
\hline Marasmus (Marasmo) & & 1 & & 1 & & \\
\hline Meningitis (Meningitis) & & & & 1 & & \\
\hline $\begin{array}{l}\text { Pleuro Pneumonia (Pleuro- } \\
\text { neumonía) }\end{array}$ & 1 & & & & & \\
\hline Pneumonia (neumonía) & & & & 1 & & \\
\hline Pulmonitis (Pulmonia) & 1 & & & 3 & 1 & \\
\hline Teething fever (Dentición) & 2 & 2 & 1 & 5 & 2 & 1 \\
\hline Tabes mesentéricas (Tuberculosis) & 14 & 13 & 3 & 16 & 13 & 4 \\
\hline None (En blanco) & & & & & & \\
\hline Totales por edad & & & & & & \\
\hline
\end{tabular}

Elaboración propia.

TABla 3

Mortalidad femenina en el Cementerio Inglés (1831-1900)

\begin{tabular}{|c|c|c|c|c|c|c|c|}
\hline \multicolumn{8}{|c|}{ Mortalidad femenina en el Cementerio Inglés (1831-1900) } \\
\hline \multicolumn{8}{|c|}{ Detalles de las defunciones por causas y edades } \\
\hline & \multirow[t]{2}{*}{ Causas de muerte } & \multicolumn{6}{|c|}{ Intervalos de edad } \\
\hline & & 10 & 20 & 30 & 40 & 50 & $60+$ \\
\hline \multirow{4}{*}{ Enfermedades infecciosas } & Croup (Crup o Difteria) & & & & & & \\
\hline & Cholera (Cólera) & & & & & & \\
\hline & $\begin{array}{c}\text { Tubercular disease } \\
\text { (Enfermedad tubercular) }\end{array}$ & & & & & & \\
\hline & Tuberculosis (Tuberculosis) & & & & & & \\
\hline $\begin{array}{l}\text { Sífilis, cáncer y otros } \\
\text { tumores malignos }\end{array}$ & Cancer (Cáncer) & & & & & & \\
\hline $\begin{array}{l}\text { Enfermedades del sistema } \\
\text { nervioso }\end{array}$ & Apoplexy (Apoplejía) & & & & & & \\
\hline
\end{tabular}




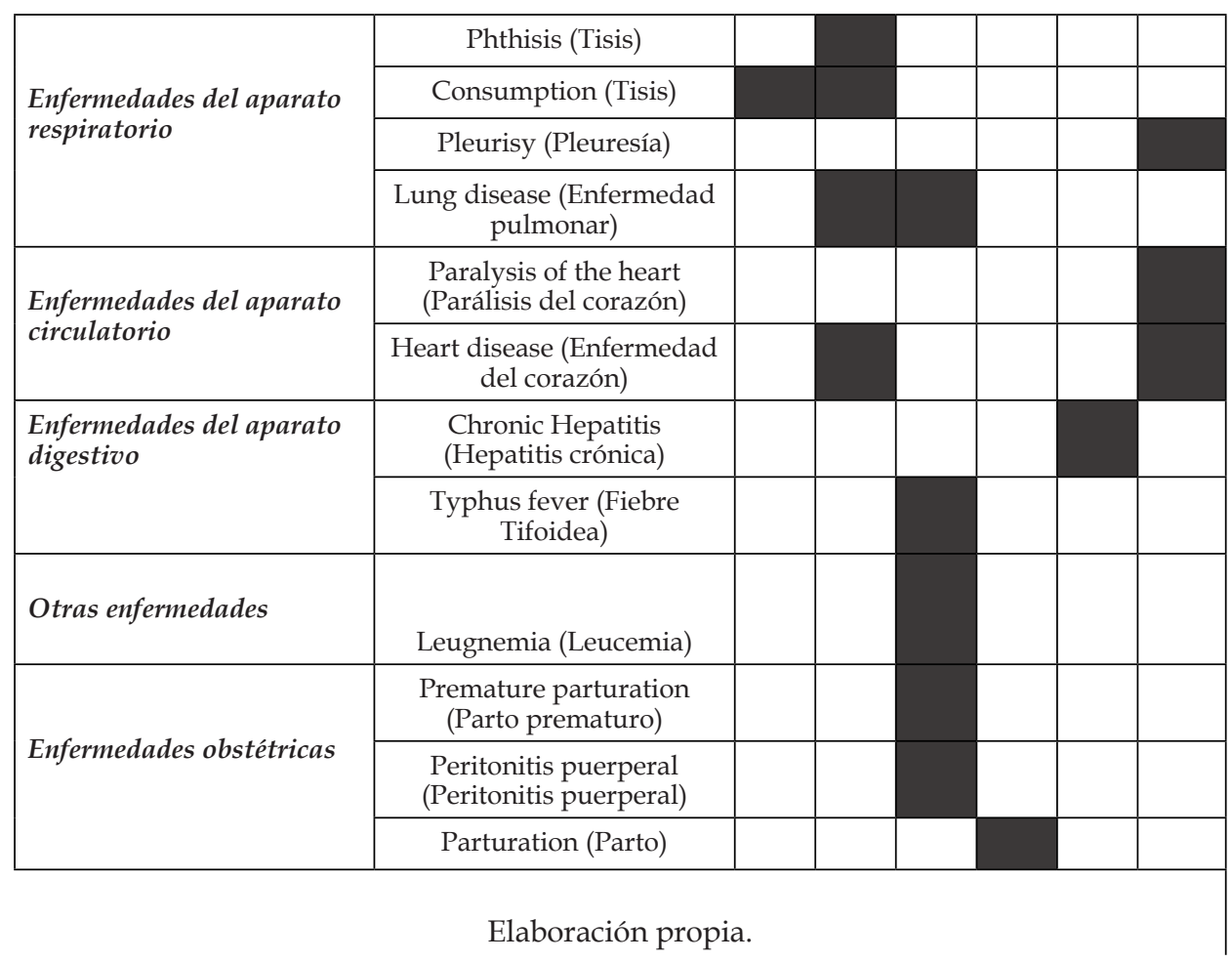


La escritura de la memoria sanitaria: el Libro de inhumaciones del cementerio inglés...

TABLA 4

Mortalidad masculina en el Cementerio Inglés (1831-1900)

\begin{tabular}{|c|c|c|c|c|c|c|c|}
\hline & Mortalidad masculina en el Cem & nteri & nglé & 1831- & & & \\
\hline & Detalles de las defunciones por causas & más & cuen & o siq & ficat & & \\
\hline & & & & Inte & los & edad & \\
\hline & Causas de muerte & 10 & 20 & 30 & 40 & 50 & $60+$ \\
\hline & Cholera (Cólera) & & & & & & \\
\hline & Cholera Morbo (Cólera Morbo) & & & & & & \\
\hline Cof $\mathrm{um}$ d d & Dysentery (Disentería) & & & & & & \\
\hline infecciosas & $\begin{array}{c}\text { Chronic Dysentery (Disentería } \\
\text { crónica) }\end{array}$ & & & & & & \\
\hline & Tuberculosis (Tuberculosis) & & & & & & \\
\hline & Putrid Fever (Fiebre Pútrida - Tifus) & & & & & & \\
\hline & Small Pox (Viruela) & & & & & & \\
\hline $\begin{array}{l}\text { Cáncer y } \\
\text { otros tumores } \\
\text { malignos }\end{array}$ & $\begin{array}{l}\text { Putrid tomour in the leg (Tumor } \\
\text { pútrido en la pierna) }\end{array}$ & & & & & & \\
\hline & Apoplexy (Apoplejía) & & & & & & \\
\hline & $\begin{array}{l}\text { Inflamation of the Brain } \\
\text { (Inflamación del cerebro - } \\
\text { Encefalitis) }\end{array}$ & & & & & & \\
\hline $\begin{array}{l}\text { Enfermedades } \\
\text { del sistema }\end{array}$ & $\begin{array}{c}\text { Rupture of a head (Ruptura de la } \\
\text { cabeza) }\end{array}$ & & & & & & \\
\hline & $\begin{array}{c}\text { Cerebral congestion (Congestión } \\
\text { cerebral) }\end{array}$ & & & & & & \\
\hline & $\begin{array}{l}\text { Softening of the Brain } \\
\text { (Ablandamiento del cerebro) }\end{array}$ & & & & & & \\
\hline & Meningitis (Meningitis) & & & & & & \\
\hline
\end{tabular}




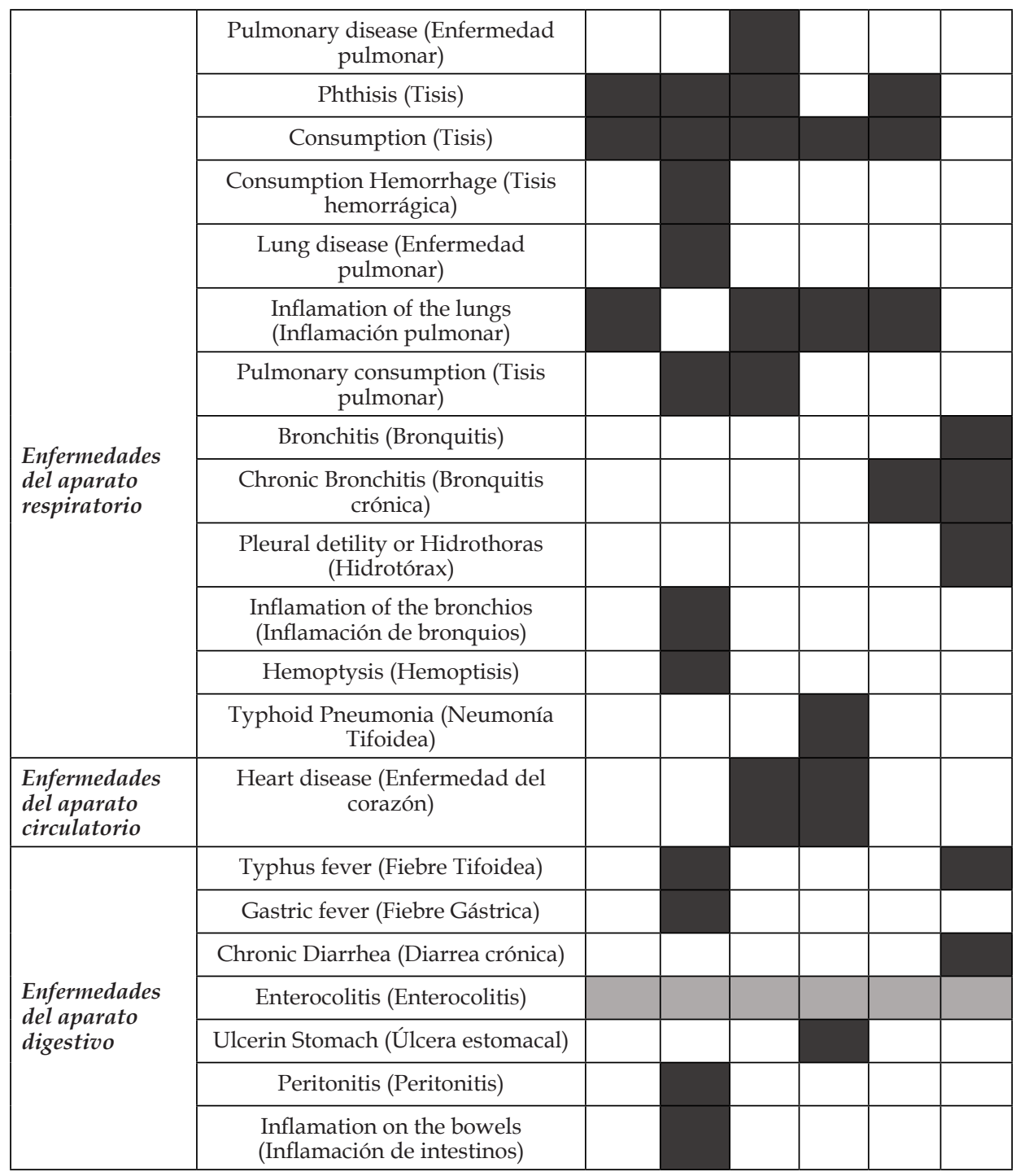


La escritura de la memoria sanitaria: el Libro de inhumaciones del cementerio inglés...

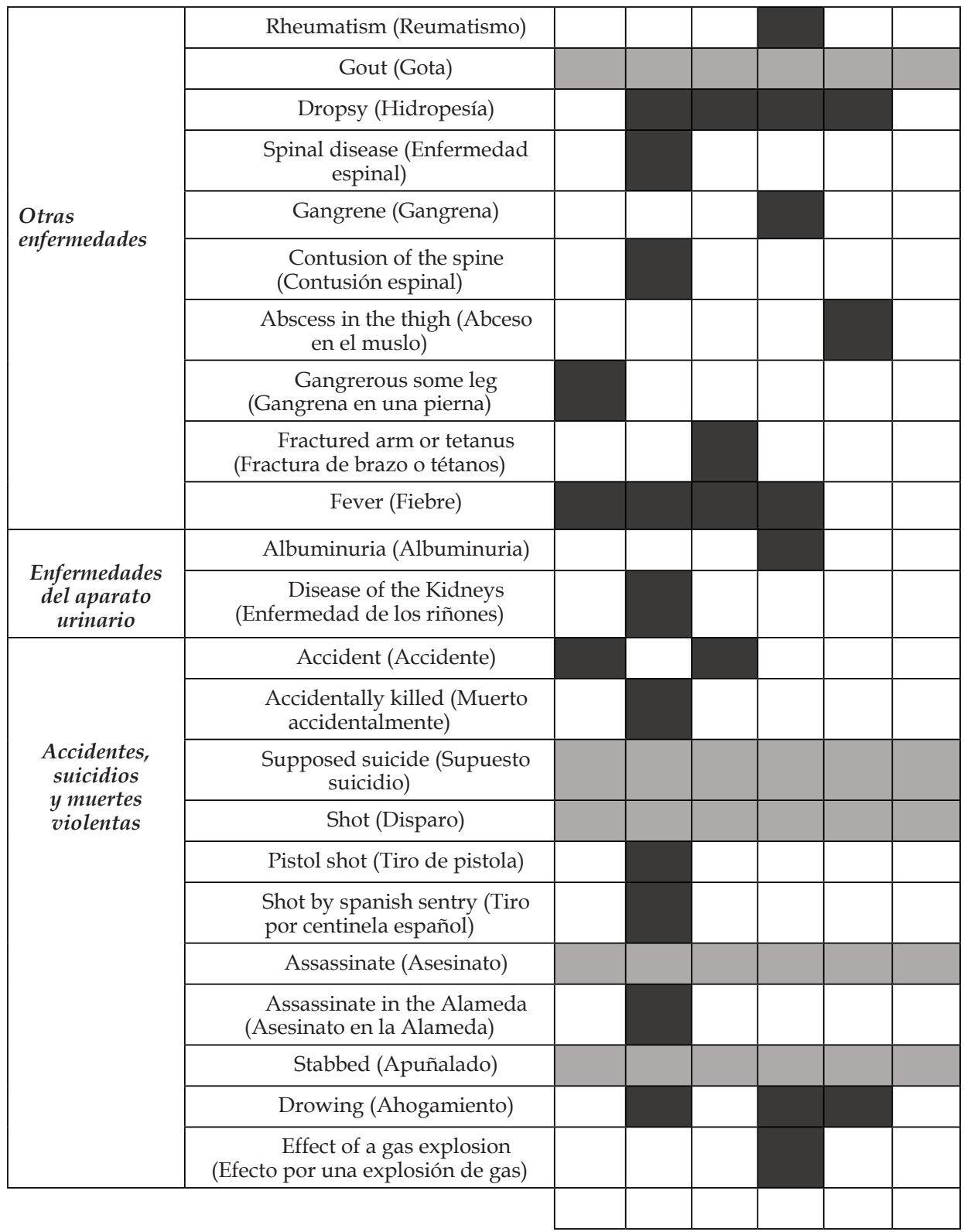

Leyenda:

\begin{tabular}{|l|l|}
\hline Intervalo de edad donde aparece con mayor frecuencia dicha causa de muerte \\
\hline Causas de muerte que carecen de edad \\
\hline
\end{tabular}

Elaboración propia. 


\section{FUENTES}

\section{Fuentes de Archivo}

Libro de inhumaciones del Cementerio Inglés de Málaga, AMM, 99-Volumen-159.

\section{Recursos Web}

Ayuntamiento de Málaga (2014). Convenio de Cesión de uso del «Libro de Registro de las Inhumaciones del Cementerio Inglés de Málaga 1831-1860» suscrito entre el Excmo. Ayuntamiento de Málaga y la Fundación del Cementerio Inglés de Málaga (22-12-2014). Ayuntamiento de Málaga, Registro de Convenios y Protocolos, Doc. 489.420, $\mathrm{n}^{\circ}$ Orden 463 (Inscripción definitiva) [en línea]. Disponible en: https://www. malaga.eu/export/sites/malagaeu/gobierno-abierto/.galleries/Convenios Protocolos/2014/4t/ID_JGL_14-08-14_CC_Cesion_uso_F_Cementerio_Ingles_-libro_de_registros_inhumaciones_del_Cementeirio_ingles_de_Ma_1931-1960u. pdf [Consulta: 03/05/2020].

Archivo Municipal de Málaga. http://archivocatalogo.malaga.eu/ms-opac/

Archivo Díaz de Escobar. http://www.museoartespopulares.com/ADE/

BuscarMuseo?ID=Documentos

Family Search. https://www.familysearch.org/es/

Graves. https://billiongraves.es/ y https://es.findagrave.com/

Plan de Ordenación General Urbanística (2010). http:/ / www.pgou.eu/Malaga.

htm

RAE. https://www.rae.es/

\section{REFERENCIAS}

Albuera Guirnaldos, A. (1998): Vida cotidiana en Málaga a fines del XIX, Ágora, Málaga.

Baudelocoue, A. C. (1831): Treatise on Puerperal Peritonitis, Elliot \& Palmer, New York.

Brel Cachón, M. P. (1999): «Comparación de los Libros Parroquiales y de los Registros Civiles. Una aportación a la validez de las fuentes demográficas a finales del siglo XIX», Boletín de la Asociación de Demografía Histórica, 17: 91-114. BRERETON, C. (1854): Lectures on the Christian Faith and Life addressed to the congregation of the Church of England at Malaga, Thomas Bosworth, London.

BRito GonZÁlez, A. D. (2015): «El descanso de la eternidad en ultramar: una breve historia del cementerio inglés de Las Palmas», Vegueta, 15: 237-256.

Burgos MadroÑERO, M. (1974): «Málaga siglos XVIII-XIX: Los extranjeros», Jábega, 7 : 49-52.

Carretero Carretero, M. (2009): «Las causas de moralidad en Burguillos del Cerro entre 1800 y 1870», Revista de Estudios extremeños, 65 (3): 1439-1464.

CARrillo, J.L. y García BalLester, L. (1980): Enfermedad y sociedad en la Málaga de los 
La escritura de la memoria sanitaria: el Libro de inhumaciones del cementerio inglés...

siglos XVIII y XIX: La fiebre amarilla (1741-1821), Universidad de Málaga, Málaga. CARrillo, M. y MendozA, J. (1834): Memoria político-médica sobre la enfermedad sufrida en esta capital en el otoño último, formada de orden de la Suprema Junta de Sanidad del Reino, (...), Viuda de Martínez Aguilar, Málaga.

Castellanos, J. (1998): «La promoción de Málaga y la idea de ciudad saludable», Dynamis. Acta hispanica ad medicinae scientiarumque historiam illustrandam, 18: 207-231.

De La Figuera, E. (2009): «Las enfermedades más frecuentes a principios del siglo XIX y sus tratamientos», en M. L. DE TORRES (coord.), Los Sitios de Zaragoza: Alimentación, Enfermedad, Salud y propaganda, Diputación de Zaragoza, Zaragoza: 151-171.

Delange Segura, D. A. (2003): Enfermedad y sociedad en Málaga. El Cólera Morbo Asiático (1833-1855), Tesis doctoral, Universidad de Málaga, Málaga.

Diaz Escovar, N. (1903): Las epidemias de Málaga: Apuntes históricos, Tipografía de «El último», Málaga.

Dunbar, S. (1862): A family tour round the coasts of Spain and Portugal during the winter of 1860-1861, William Blackwood and sons, Edinburg and London.

FORD, R. (1845): Hand-book for travellers in Spain. Describing the country and cities, the natives and their manners with notices on Spanish history, John Murray, London.

Francis, D. J. T. (1853): Change of Climate Considered as a Remedy in Dyspeptic, Pulmonary, and other chronic affections; with an account of the most elegible places of residence for invalids in Spain, Portugal, Algeria, Etc., at different seasons of the year and an appendix on the mineral springs of the Pyrenees, Vichy, and Aix les Bains (...), John Churchill, London.

Freire López, A. (2012): «España y la literatura de viajes en el siglo XIX», Anales, 24: 67-82.

GAlante Gómez, F. J. (1990): «Los cementerios: otra lectura de la ciudad burguesa», en F. Morales (ed.), Actas del vil Coloquio de Historia Canario-Americana, vol. II, Cabildo Insular de Gran Canaria, Las Palmas de Gran Canaria: 602-624.

Galende Diaz, J. y Ávila SeOANe, N. (2011): «Los libros sacramentales de defunción del Madrid moderno», en IX Jornadas Científicas sobre Documentación: La muerte y sus testimonios escritos, Madrid: 125-169.

Girón Irueste, E. y Arenas Gómez, A. (2003): «La imagen del Cementerio Inglés de Málaga en los viajeros extranjeros: La mirada del otro», en M. B. VILLAR García y P. Pezzi Cristóbal (eds.), Los extranjeros en la España Moderna. Actas del I Congreso Internacional, Tomo II, Málaga: 359-368.

Grice-Hutchinson, M. (1989): El Cementerio Inglés de Málaga y otros estudios, Universidad de Málaga, Málaga.

Guillén Robles, F. (1983): Historia de Málaga y su provincia, Vol. II, Arguval, Málaga. Haverty, M. (1844): Wanderings in Spain in 1843, T. C. Newby, London.

Heredia Flores, V. y Aguilera García, J. (2019): «El Cementerio Inglés y el mar», Cuadernos del Rebalaje, 25, ABJ, Málaga.

JimÉNEZ Quintero, J. A. (2009): «Industria malagueña, S. A.», Jábega, 100: 115-124.

Krauel Heredia, B. (1986): Viajeros británicos en Andalucía: de Christopher Hervey a 
Richard Ford (1760-1845), Universidad de Málaga, Málaga.

Lara García, M. P. (2005): «Historia del Hospital Noble de Málaga (1865-2004)», Isla de Arriarán, 25:189-215.

LEE, E. (1854): Notes of Spain; with a Special Account of Malaga and its Climate, Hope \& Co, London.

Mateo Avilés, E. (2001): «La tragedia de la Gneisenau y su impacto en la Málaga del momento», Isla de Arriarán, 17: 163-176.

Marchant Rivera, A. (2004): "Moradas de la "escritura última": Fuentes documentales para la historia del Cementerio Inglés de Málaga», Revista de Historiografía, 1: 161-166.

Marchant Rivera, A. (coord.) (2005): El Cementerio Inglés de Málaga: Tumbas y epitafios, Spicum, Málaga.

Marchant Rivera, A. (2012): «El Cementerio Inglés de Málaga: un jardín de historia cercano al mar», Uciencia, 9: 50-52.

Marchant Rivera, A. (2014): Estudios sobre el Cementerio Inglés de Málaga, Libros Encasa, Málaga.

Marchant Rivera, A. y Barrena Gómez, A. (en prensa): «Documentos para la memoria sanitaria: los partes de enfermos del Hospital Noble de Málaga, año 1874», Documenta et Instrumenta, 19.

Martínez y Montes, V. (1852): Topografía Médica de Málaga, Ramón Franquelo, Málaga.

More Madden, T. (1874): On Change of Climate. In the Treatment of Chronic Diseases, Especially consumption, a guide por travellers in pursuit of health to the Southern winter resorts of Europe and Africa for the south of France, Spain, Portugal, Italy, Algeria, The Mediterranean Islands, Egypt Ec., T.C. Newby, London.

Pá́z Galán, E. (1980): "Naufragio de la fragata alemana “Gneisenau” y el puente del Santo Domingo», Jábega, 29: 3-11.

Prothero, R. E. (1905): The letters of Richard Ford 1797-1858 (...), John Murray, London.

RAMírez SÁnCHEZ, M. (2016): Historias en la piedra: La escritura última en los cementerios ingleses de Canarias, Dykinson, Madrid.

Robles GonzÁlez, E. (2002): La transición de la mortalidad infantil y juvenil en las comarcas meridionales valencianas 1838-1960, Tesis doctoral, UNED, Madrid.

Rodríguez Marín, F.J. (2005): «Patrimonio y ciudad. Valores artísticos y culturales en el Cementerio Inglés de Málaga: Entre la magnificencia y el deterioro», Isla de Arriarán, 25: 23-58.

RuIz MAs, J. (2008): «Médicos-viajeros y viajeros-convalecientes de habla inglesa en la España mediterránea durante el siglo XIX», Epos, 24: 175-189.

TARRÉs, S.; Moreras, J. (2012): «Patrimonio cultural funerario. Los cementerios de las minorías religiosas en España», en B. SANTAMARÍA (coord.), Geopolíticas patrimoniales de culturas, naturalezas e inmaterialidades: una mirada etnográfica, Germanía, Valencia: 267-283.

Tenison, L. (1853): Castile and Andalucia, Richard Bentley, London.

Villar García, M.B. (1982): «Los extranjeros en Málaga en el siglo XVIII (Resumen de una investigación (1)» en Baetica. Estudios de Arte, Geografía e Historia, 5: 
La escritura de la memoria sanitaria: el Libro de inhumaciones del cementerio inglés...

205-214.

VV.AA (1859): The American and foreign Christian Union - "the love of christ constraineth us", Vol. X, The American and foreign Christian Union, New York.

VV.AA (1881): El Defensor de Granada: diario político independiente, 2, 297. 